\title{
Exercise Is Medicine, but Does Not Need To Be Prescribed by a Physician: Time to Recognize the Role and Expertise of the Clinical Exercise Professional
}

Zoe L. Saynor, PhD | Anthony I. Shepherd, PhD, MSc

PRiMER. 2022;6:4.

Published: 2/17/2022 | DOI: 10.22454/PRiMER.2022.950894

\section{To the Editor:}

We read the recent work published by Adedokun and colleagues. ${ }^{1}$ We would argue that exercise is medicine, but does not need to be prescribed by a physician, and that it's time to recognize the role and expertise of the clinical exercise professionals and change the perspective, toward a recognized inclusion within the multidisciplinary team (MDT). The MDT is a standard part of health care and is essential for effective clinical management of many long-term conditions. There is a need to recognize the important role of the clinical exercise practitioner and the years of training, expertise and skills that these practitioners bring to the health care system. The incorrect and interchangeable use of physical activity and exercise terminology within this article are testament to this. As outlined in a recent United Kingdom-based, forward-thinking document, ${ }^{2}$ any services managing age-related and chronic diseases need a highly qualified exercise health care professional to be an integral part of the care pathway and MDT.

Exercise testing, assessment, interpretation, prescription, delivery, and outcomes evaluation for individuals with chronic and complex conditions requires a specialist knowledge base and expertise. ${ }^{3}$ Clinical exercise professionals conduct screening and assessments, apply independent clinical and scientific reasoning to develop safe and effective interventions that target the specific needs of the individual. ${ }^{4}$ Interventions delivered by these individuals are evidence based, include specific exercise prescriptions, health and physical (in)activity education, as well as support for lifestyle modification and behavior change.

The key messaging in this study is disappointing, because many of us specialising in clinical exercise physiology from across the globe view the American model as world leading. Specifically, qualified exercise professionals with formal registration and accreditation (certified exercise physiologists) are already established in the United States, with the American College of Sports Medicine (ACSM) being the accrediting body for people in countries outside the United States without such an accreditation route. We believe that the focus should be on educating medical students about not only the physical activity guidelines, but also the services that are already in place to ensure appropriate signposting and referrals to the best health care specialist trained in this area, which will ultimately lead to the best clinical outcomes.

In 2013, more than half of the physicians trained in the United States received no formal education in physical activity, ${ }^{3}$ leading to a call to reverse this situation by The Bipartisan Policy Center, ACSM, and Alliance for a Healthier Generation in 2014. In this study by Adedokun and colleagues, many medical students were indeed 
aware of the general physical activity guidance and national recommendations for aerobic exercise. There should be a change in focus and realization that most physicians will not and perhaps need not be experts in physical activity and exercise testing and training. There is an established profession and they should be recognised and used appropriately.

\section{Corresponding Author}

Zoe L. Saynor, PhD

Saynor, School of Sport, Health and Exercise Science, Faculty of Science and Health, University of Portsmouth, Hampshire, UK, PO1 2ER. Tel: +44 (0)2392 843080

zoe.saynor@port.ac.uk

\section{Author Affiliations}

Zoe L. Saynor, PhD - Physical Activity, Health and Rehabilitation Thematic Research Group, School of Sport, Health and Exercise Science, Faculty of Science and Health, University of Portsmouth, Portsmouth, UK Anthony I. Shepherd, PhD, MSc - Physical Activity, Health and Rehabilitation Thematic Research Group, School of Sport, Health and Exercise Science, Faculty of Science and Health, University of Portsmouth, Portsmouth, UK

\section{References}

1. Adedokun CA, Curles WG, DeMaio EL, Asif IM. Analysis of American medical students' knowledge of physical activity recommendations. PRiMER. 2021;5:31. doi:10.22454/PRiMER.2021.249084

2. Jones $\mathrm{H}$, George KP, Scott $A$, et al. Charter to establish clinical exercise physiology as a recognised allied health profession in the UK: a call to action. BMJ Open Sport Exerc Med. 2021;7(3):e001158. doi:10.1136/bmjsem-2021-001158

3. Cardinal BJ, Park EA, Kim M, Cardinal MK. If exercise is medicine, where is exercise in medicine? Review of US medical education curricula for physical activity-related content. J Phys Act Health. 2015;12(9):1336-1343. doi:10.1123/jpah.2014-0316

4. Franklin B, Fern A, Fowler A, Spring T, Dejong A. Exercise physiologist's role in clinical practice. $\mathrm{Br} \mathrm{J}$ Sports Med. 2009;43(2):93-98. doi:10.1136/bjsm.2008.055202

Copyright $(2022$ by the Society of Teachers of Family Medicine

\section{Related Content}

Adedokun CA, Curles WG, DeMaio EL, Asif IM. Analysis of American Medical Students' Knowledge of Physical Activity Recommendations. PRiMER. 2021;5:31. https://doi.org/10.22454/PRiMER.2021.249084 